\title{
OPEN KNOWLEDGE MAPS: VISUELLE LITERATURSUCHE BASIEREND AUF DEN PRINZIPIEN VON OPEN SCIENCE
}

\section{von Peter Kraker, Maxi Schramm und Christopher Kittel}

Zusammenfassung: Die Wissenschaft befindet sich in einer Auffindbarkeitskrise. Obwohl durch die Open Access-Bewegung Forschungsergebnisse besser zugänglich geworden sind, wird ein signifikanter Teil der Outputs nicht nachgenutzt. Einen großen Anteil an der Krise haben die Tools, die für die Literatursuche verwendet werden. Angesichts von drei Millionen Veröffentlichungen pro Jahr sind klassische Ansätze, wie etwa listenbasierte Suchmaschinen, nicht mehr ausreichend. Open Knowledge Maps hat es sich zum Ziel gesetzt, die Auffindbarkeit wissenschaftlichen Wissens zu verbessern. Dafür betreibt die gemeinnützige Organisation aus Österreich die weltweit größte visuelle Suchmaschine für Forschung. Das Grundprinzip besteht darin, Wissenslandkarten für die Literatursuche zu nutzen. Diese geben einen Überblick über ein Forschungsfeld und ermöglichen so einen schnelleren Einstieg in die Literatur. Open Knowledge Maps basiert auf den Prinzipien von Open Science: Inhalte, Daten und Software werden unter einer freien Lizenz veröffentlicht. Dadurch entsteht eine offene, wiederverwendbare Infrastruktur; Lock-In-Effekte, wie sie bei proprietären Systemen auftreten, werden vermieden. Open Knowledge Maps arbeitet seit Beginn eng mit Bibliotheken und Bibliothekarlnnen als ExpertInnen für Wissensorganisation und -verwaltung zusammen. Im Rahmen eines konsortialen Fördermodells werden Bibliotheken nun eingeladen, das System stärker mitzugestalten - unter anderem bei wichtigen Zukunftsthemen wie der besseren Auffindbarkeit von Datensätzen.

Schlagwörter: Open Science; Auffindbarkeit; Literatursuche; Wissenslandkarten; Visualisierung; Offene Infrastrukturen; Nachhaltigkeit; konsortiale Finanzierung

\section{OPEN KNOWLEDGE MAPS: VISUAL DISCOVERY BASED ON THE PRINCIPLES OF OPEN SCIENCE}

Abstract: There is a discoverability crisis in research. A significant share of research
outputs are not reused, even though publications are more easily accessible thanks
to the open access movement. One of the main reasons for the crisis are the tools
that we use for discovery. Classic list-based approaches are not suitable any more
for the three million papers that are published each year. Open Knowledge Maps is
dedicated to improving the visibility of scientific knowledge. To this end, the charitable
nonprofit organisation operates the largest visual search engine in the world. Our ap-
proach is to use knowledge maps for discovery. Knowledge maps provide an instant 
overview of a scientific field and thus enable users to get acquainted with the literature much more quickly. Open Knowledge Maps is based on the principles of open science: content, data, and software are shared under an open license. This creates an open infrastructure, avoiding the lock-in effects of proprietary systems. Since the beginning, Open Knowledge Maps has worked together closely with libraries and librarians as the experts for knowledge organisation and management. As part of a consortial funding model, we now invite libraries to co-create the system with us - this includes important upcoming topics, such as the discoverability of research data.

Keywords: Open Science; Discoverability; Literature Search; Knowledge Maps; Visualization; Open Infrastructures; Sustainability; Consortial Funding

DOI: https://doi.org/10.31263/voebm.v72i2.3202

(c) Peter Kraker, Maxi Schramm, Christopher Kittel

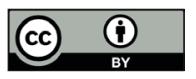

Dieses Werk ist lizenziert unter einer

Creative-Commons-Lizenz Namensnennung 4.0 International

\section{Inhalt}

1. Einleitung

2. Dark Knowledge und die Krise der Auffindbarkeit

3. Probleme der klassischen Literatursuche

4. Open Knowledge Maps: mit Wissenslandkarten zum schnellen Überblick

5. Open Knowledge Maps lebt Open Science

6. Aus Österreich für die ganze Welt

7. Nachhaltigkeit durch ein konsortiales Fördermodell

8. Exkurs: die problematische Fördersituation gemeinnütziger Open Source-Initiativen

9. Starke Partnerschaft mit Bibliotheken

10. Ausblick: Kollaborative Literaturrecherche 


\section{Einleitung}

In Westafrika brach 2014 die schlimmste Ebola-Epidemie der Geschichte aus. Die Epidemie traf mit Liberia, Sierra Leone und Guinea drei der ärmsten Länder der Welt und konnte erst nach drei Jahren und über 11.000 Toten für beendet erklärt werden (Wikipedia 2019). Einer der wichtigsten Gründe für die Schwere des Ausbruchs war, dass Gesundheitsbehörden rund um die Welt davon ausgingen, dass das Ebola-Virus in der Region nicht endemisch ist, also dass es dort nicht heimisch ist. Dadurch waren sowohl die Gesundheitsbehörden in den betroffenen Ländern als auch internationale Organisationen wie die WHO unvorbereitet auf den Ausbruch, weswegen dieser solche Ausmaße annehmen konnte.

Doch wie eine Untersuchung, veröffentlicht in der New York Times, ergab (Dahn et al. 2015), war seit mehr als dreißig Jahren bekannt, dass Ebola in Westafrika heimisch ist. In einem Paper in den Annals of Virology aus dem Jahr 1982 wurde Ebola erstmals als endemisch in Westafrika beschrieben und in den Folgejahren kamen mehrere wissenschaftliche Artikel zu dem gleichen Ergebnis. Die Ebola-Epidemie hätte somit auf der Basis von jahrzehntealten Forschungsergebnissen verhindert bzw. stark eingedämmt werden können. Doch dieses Wissen blieb den Gesundheitsbehörden verborgen.

Dabei handelte es sich nicht um ein Problem der Zugänglichkeit: die betroffenen Länder haben aufgrund ihres Status als Entwicklungsländer über spezielle Förderprogramme wie z.B. Research4Life ${ }^{1}$ kostenlosen Zugang zu den entsprechenden Artikeln und internationale Organisationen wie die WHO und das Institut Pasteur verfügen über Subskriptionen zu den entsprechenden Zeitschriften. Es handelte sich vielmehr um ein Problem der Auffindbarkeit: obwohl die relevanten Stakeholder Zugriff auf die Artikel gehabt hätten, konnten sie das für sie relevante Wissen nicht entdecken. Ein Teil des Problems liegt sicher darin begründet, dass die angesprochenen Artikel von europäischen Wissenschaftlerlnnen ohne westafrikanische Ko-Autorlnnen verfasst und in europäischen Zeitschriften veröffentlicht wurden. Dies erklärt aber noch nicht, warum internationale Organisationen mit Wurzeln im globalen Norden dieses Wissen ebenso verborgen blieb. Das Institut Pasteur ist sogar Herausgeber von Annals of Virology, jener Zeitschrift, in der das oben besprochene Paper aus dem Jahr 1982 erschienen ist.

\section{Dark Knowledge und die Krise der Auffindbarkeit}

Das Phänomen, dass eigentlich öffentliches Wissen im Verborgenen bleibt, hat seit kurzem einen Namen: "Dark Knowledge” (Jeschke et al. 2019). 
Der Begriff, geprägt von einer Gruppe um den Berliner Ökologen Jonathan Jeschke und den nunmehrigen Präsidenten des FWF, Klement Tockner, bezeichnet Wissen, welches nicht gefunden und nicht wieder verwendet werden kann. Kurz gesagt: man sieht den Wald vor lauter Bäumen nicht.

Open Science wird oft als Gegenmittel zu Dark Knowledge gesehen. Zu einem gewissen Grad stimmt das auch: durch Open Access wurde die Zugänglichkeit zu wissenschaftlichen Artikeln dramatisch erhöht. Doch bei der Auffindbarkeit hat sich wenig getan. Mit drei Millionen Veröffentlichungen pro Jahr, Tendenz steigend (Johnson et al. 2018), wird die Literatursuche zunehmend schwieriger. Klassische Ansätze, wie etwa listenbasierte Suchmaschinen, sind angesichts der Flut neuen Wissens nicht ausreichend. Dies spiegelt sich auch in den Zahlen zur Wiederverwendung wider: je nach Disziplin werden zwischen $7 \%$ und $38 \%$ von wissenschaftlichen Publikationen nie zitiert, bei jenen ohne disziplinäre Zuordnung $63 \%$ (Nicolaisen \& Frandsen 2019).

Bei Datensätzen steigt die Uncitedness (Nicht-Zitierheit) sogar auf bis zu $85 \%$ an (Peters et al. 2016). Besonders problematisch ist es auch beim Transfer in die Praxis; selbst in anwendungsorientierten Disziplinen wie der Medizin wird nur eine Minderheit der Forschungsergebnisse jemals in der klinischen Praxis angewandt, und wenn, dann mit großer Verzögerung (Brownson et al. 2006).

All dies zeigt, dass wir uns in einer veritablen Auffindbarkeitskrise befinden. Diese Krise hat einen negativen Einfluss auf die Effizienz und Effektivität von Wissenschaft, da die Kommunikation innerhalb der wissenschaftlichen Community und der Transfer zur Praxis dadurch entscheidend behindert werden.

\section{Probleme der klassischen Literatursuche}

Einer der Gründe für die Krise der Auffindbarkeit ist ein Mangel an Innovationen bei geschlossenen und proprietären Suchmaschinen. Ein Beispiel dafür ist Google Scholar. Als Google Scholar vor etwa fünfzehn Jahren auf den Markt kam, war es eine bahnbrechende Literatursuchmaschine. Der Bestand an wissenschaftlicher Literatur hat sich inzwischen allerdings nahezu vervierfacht (Bornmann \& Mutz 2015). In dieser Zeit haben sich Funktionsweise und Interface von Google Scholar allerdings nur geringfügig verändert. Dies zeigt sich im Vergleich der Suchresultate aus den Jahren 2010 und 2019 (siehe Abbildungen 1 und 2). Such- und Filterfunktionen, wie auch die Darstellung der Ergebnisse sind nahezu unverändert. Im Gegensatz dazu wurden etwa die Google Suchresultate in den letzten Jahren immer stärker mit Ele- 


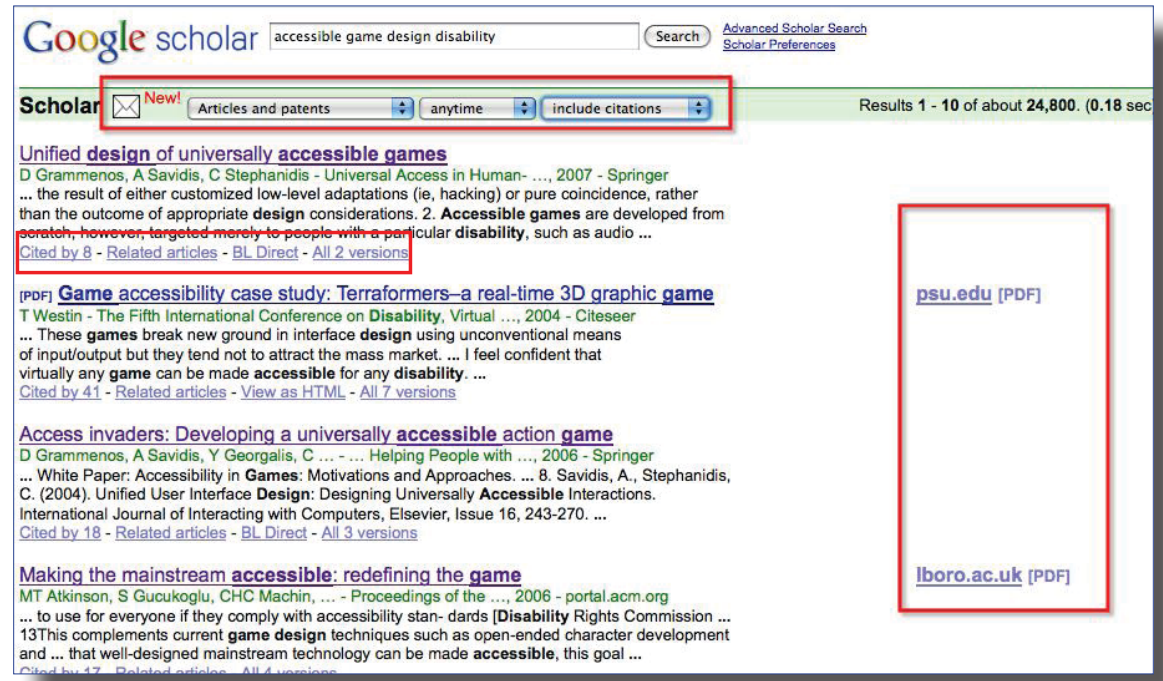

Abb. 1: Google Scholar im Jahr 2010. Abbildung von Tony Hirst, CC BY 2.0. Hervorhebung relevanter Funktionalitäten durch den Ersteller der Abbildung und die Autorlnnen.

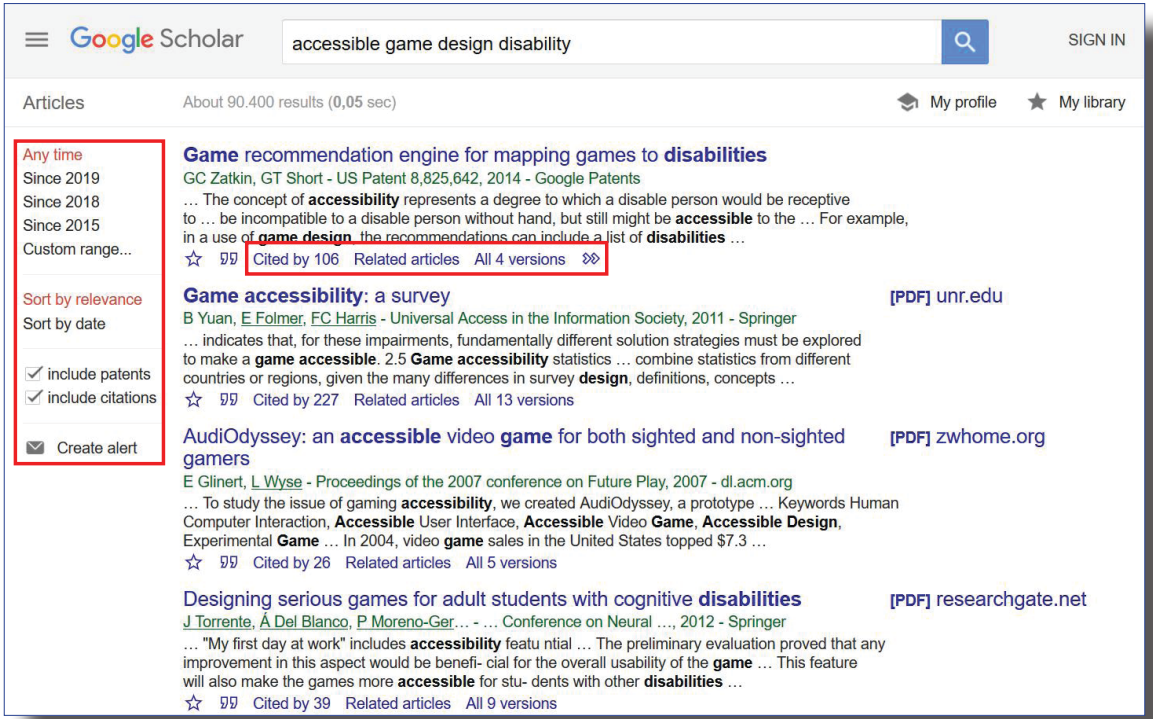

Abb. 2: Google Scholar im Jahr 2019. Screenshot. Hervorhebung relevanter Funktionalitäten durch die Autorlnnen. 
menten wie Bildern, Karten, verwandten Suchen und und Einträgen aus dem Knowledge Graph kontextualisiert. Hier wurde seitens Google nicht genug in Google Scholar investiert, um mit diesem Wachstum Schritt zu halten. Daher ist Google Scholar heutzutage von sehr begrenztem Nutzen und hilft Forscherlnnen bei der Suche nach relevanten Dokumenten nur noch bedingt.

Dies trifft auch auf andere Anbieter zu: in einer Umfrage unter 107 mehrheitlich österreichischen Forschenden und Studierenden, durchgeführt mit der FH Joanneum Graz, zeigte sich nur etwa ein Drittel zufrieden mit dem derzeitigen Angebot an akademischen Literatursuchtools (siehe Abbildung 3). Die Respondenten nutzen dabei eine Vielzahl an Tools: generische Web-Suchmaschinen (84,1\%), akademische Web-Suchmaschinen (74,8\%), Portale wie ResearchGate und Academia.edu und Bibliothekskataloge (jeweils 40,2\%), Datenbanken wie MLA oder JSTOR (31,8\%), sowie andere Literatursuchtools (25,0\%). Insgesamt nutzten $88,8 \%$ Literatursuchtools aus mehreren Kategorien, $60,7 \%$ sogar Tools aus drei oder mehr Kategorien. Dennoch empfanden nur $34,4 \%$ es als einfach, relevante Publikationen zu identifizieren und lediglich ein Drittel der Respondenten befand es als einfach, eine Suchanfrage zu einem unbekannten Thema oder in einem unbekannten Feld zu formulieren. Eine überwiegende Mehrzahl der Respondenten empfindet es auch als schwierig, einen Überblick über ein Thema mithilfe von akademischen Literatursuchtools zu bekommen und ist der Meinung, Literatursuche dauere zu lange (Ablehnung der relevanten negativ formulierten Statements zu 31,4\% bzw. 28,6\%). Die Befragung wurde in der Open Science-Community und unter Studierenden der FH Joanneum Graz durchgeführt. Die Ergebnisse müssen dementsprechend noch in einer größeren, repräsentativen Studie überprüft werden. Dennoch weisen die vorläufigen Resultate auf entsprechende Defizite bei bestehenden Tools hin.

1. Ich empfinde es als einfach, relevante Publikationen mit ALST zu identifizieren

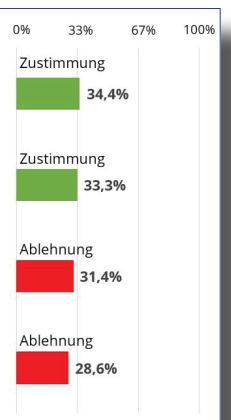

Abb. 3: Ergebnisse einer Umfrage zu ALST (Akademischen Literatursuchtools). Fragen aus dem Englischen übersetzt. $\mathrm{n}_{\text {Frage } 1}=105, \mathrm{n}_{\text {Frage } 2,3,4}=102$. 
Dieser Mangel an Innovation wäre nun kein Problem, wenn andere Tools auf den Google Scholar-Index aufbauen könnten. Dies ist jedoch leider nicht möglich, da der Index nicht wiederverwendet werden darf, nach dem Motto: „Anschauen erlaubt, weitergeben verboten“. Innovatoren in diesem Markt müssen zunächst einen eigenen Index aufbauen - ein mühsames Unterfangen, welches durch Googles zahlreiche Sondervereinbarungen mit Verlagen nicht leichter wird. Andere kommerzielle Anbieter wie Elsevier (Scopus) und Clarivate (Web of Science) verfolgen im Übrigen die gleiche Strategie, wenn es um Wiederverwendung der Indizes geht, auch wenn sich die Geschäftsmodelle unterscheiden. So monetarisiert Google die Nutzerlnnendaten über personalisierte Werbung, wohingegen Elsevier und Clarivate vorwiegend Lizenzgebühren einheben.

Darüber hinaus bietet Google Scholar nur unstrukturierte Ergebnislisten, mit jeweils 10 Ergebnissen pro Seite. Dies funktioniert sehr gut, wenn der Informationsbedarf bereits genau definiert ist. Will man sich allerdings erst einmal einen Überblick über ein Forschungsthema verschaffen, braucht man auf diese Weise Wochen, wenn nicht Monate, bevor man die wichtigsten Themen, Veröffentlichungsorte und Autorlnnen identifiziert hat. Das dauert zu lange - vor allem, wenn eine Katastrophe in Form einer öffentlichen Gesundheitsnotlage eintritt.

Dies alles zeigt, dass dringend neue Lösungsansätze für die Literatursuche benötigt werden, um von den Ergebnissen der Open Access-Bewegung besser profitieren zu können.

\section{Open Knowledge Maps: mit Wissenslandkarten zum schnellen Überblick}

Open Knowledge Maps hat es sich zum Ziel gesetzt, die Auffindbarkeit wissenschaftlichen Wissens zu verbessern. Ziel ist es, "Dark Knowledge" für Wissenschaft und Gesellschaft wieder sichtbar zu machen. Die gemeinnützige Organisation mit Sitz in Wien arbeitet dabei mit einem internationalen Netzwerk, bestehend aus Teammitgliedern, Advisorn und Partnerorganisationen, zusammen ${ }^{2}$. Um ihre Mission zu erfüllen, betreibt Open Knowledge Maps die weltweit größte visuelle Suchmaschine für Forschung. Das frei zugängliche Tool ermöglicht verschiedenen Stakeholdern, wissenschaftliche Inhalte zu entdecken, zu untersuchen und wieder zu verwenden. Zu den NutzerInnen gehören WissenschaftlerInnen, Studierende, BibliothekarInnen, PädagogInnen, WissenschaftsjournalistInnen und Fachkräfte aus verschiedenen Disziplinen. Open Knowledge Maps erhöht die Sichtbarkeit von Inhalten verschiedener 
Quellen unter anderem von Bibliotheken, Repositorien, Fördergebern und Verlagen.

Das Grundprinzip besteht darin, sogenannten "knowledge maps", zu deutsch „Wissenslandkarten“, für die Literatursuche zu nutzen. Wie Abbildung 4 illustriert, haben diese einige Vorteile gegenüber rein listenbasierten Suchmaschinen: sie bieten einen Überblick über ein Forschungsfeld, indem sie die Hauptthemen des wissenschaftlichen Forschungsgebietes auf einen Blick anzeigen. Zudem sind den einzelnen Themen bereits relevante Publikationen zugeordnet. Dadurch können Interessierte direkt in ein Thema einsteigen. Durch die Benennung der Themenfelder wird zudem im gleichen Schritt die Terminologie aufgebaut, oft der langwierigste Teil einer Literaturrecherche.

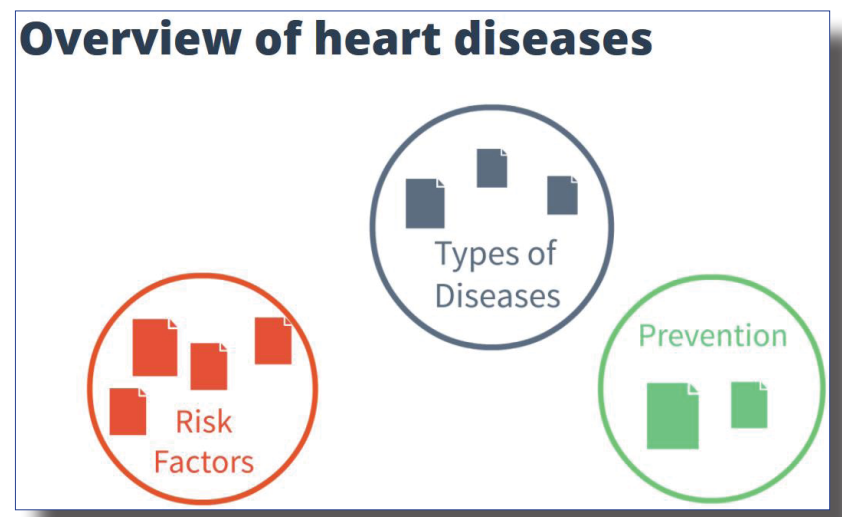

Abb. 4: Vereinfachtes Beispiel einer Wissenslandkarte für den Bereich „Herzerkrankungen“

Basierend auf dieser Idee bietet Open Knowledge Maps auf der Website openknowledgemaps.org einen frei zugänglichen Service an, mit dem Wissenslandkarten zu Forschungsthemen aller Disziplinen erstellt werden können. Nutzerlnnen können dabei zwischen zwei Datenbanken wählen: zum einen BASE (Bielefeld Academic Search Engine), der Suchdienst der Universitätsbibliothek Bielefeld, mit mehr als 140 Millionen wissenschaftlichen Dokumenten aus allen Disziplinen und zum anderen PubMed, die große naturwissenschaftliche Datenbank der NIH mit Fokus auf Biomedizin, welche über 28 Millionen Referenzen indiziert.

Eine beispielhafte Wissenslandkarte für den Begriff "digital education" ist in Abbildung 5 zu sehen. Wie im Beispiel weiter oben werden die verschiedenen Themen als Kreise angezeigt. Bei Klick auf eine der Bubbles werden die zugeordneten Publikationen präsentiert. Open Knowledge Maps ver- 
steht sich dabei als ein Interface zum gesamten wissenschaftlichen Wissen, offen und geschlossen; Open Access-Artikel werden jedoch gekennzeichnet und können direkt im Interface gelesen werden. Zudem ist mit Hypothesis auch ein eigenes Annotationsservice für Open Access-Artikel eingebunden.

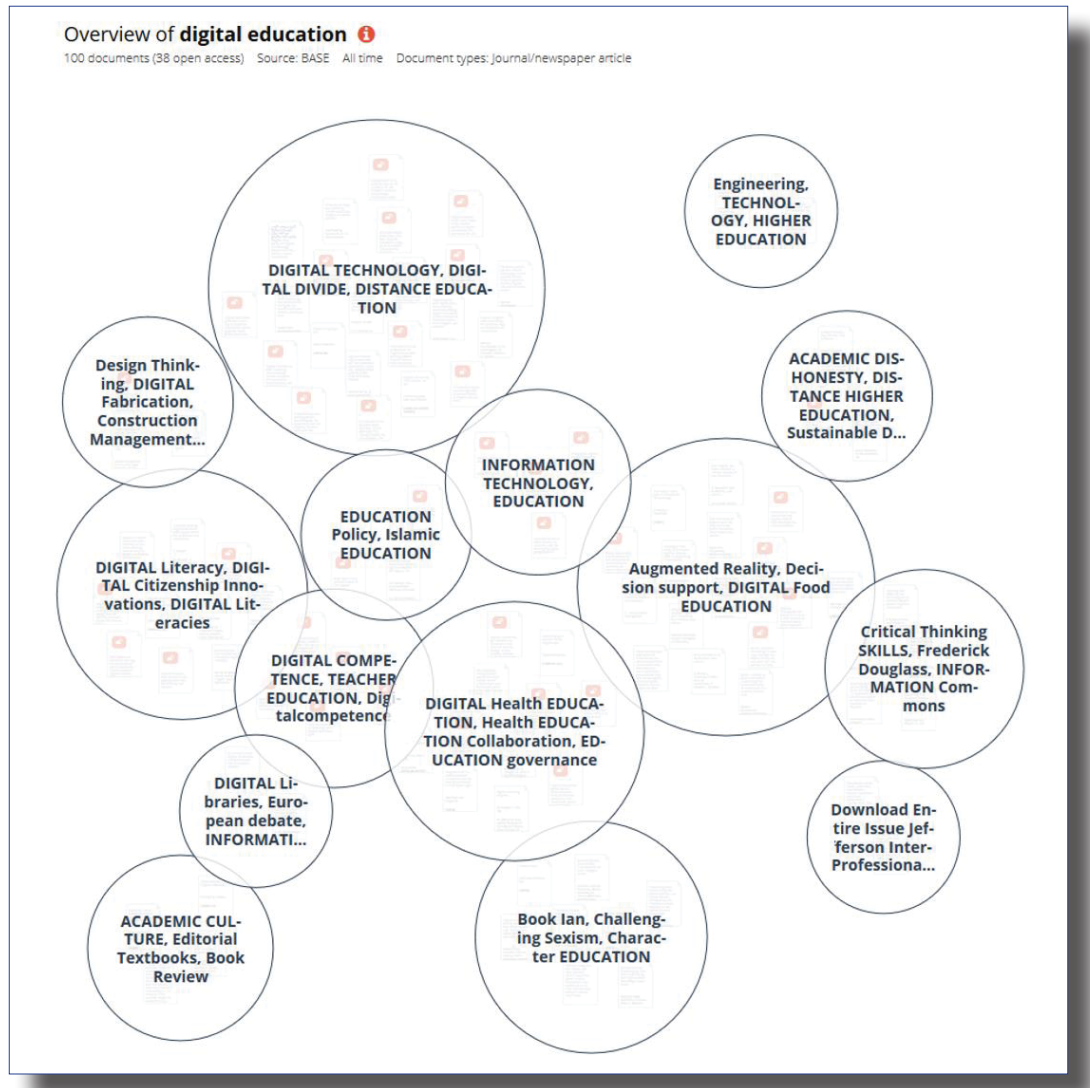

Abb. 5: Wissenslandkarte für den Begriff "digital education". Quelle: https://openknowledgemaps.org/map/c8fe7a11ca29a8b8580e5612fcebc42a

Für die Erstellung der Wissenslandkarte nutzt Open Knowledge Maps derzeit die 100 relevantesten ${ }^{3}$ Dokumente für den Suchbegriff aus der jeweiligen Datenbank. Anschließend wird eine Repräsentation der Karte auf der Basis der Wortähnlichkeit zwischen den Metadaten der Dokumente berechnet. Das heißt: je mehr Worte zwei Dokumente in Titel, Abstract, Autoren und Zeitschrift gemeinsam haben, desto näher werden sie auf der Karte angeordnet und desto wahrscheinlicher werden sie einem Bereich 
zugeordnet. Die Repräsentation der Wissenslandkarte wird dann an das nutzerseitige Frontend ausgeliefert. Dieses besteht aus einer webbasierten, interaktiven Visualisierung, welche dem Prinzip von Shneiderman (1996) "Overview first, zoom and filter, then details-on-demand" folgt. Weitere Informationen über die technische Infrastruktur finden sich in einem Artikel publiziert in 027.7 (Kraker et al. 2016).

\section{Open Knowledge Maps lebt Open Science}

Im Gegensatz zu Google Scholar wird Open Knowledge Maps basierend auf den Prinzipien von Open Science als offene, wiederverwendbare Infrastruktur entwickelt. Dies bedeutet, dass die gesamte Software als Open Source unter einer freien Lizenz entwickelt wird (Kraker et al. 2019). Die Wissenslandkarten selbst sind mit einer Creative Commons Namensnennung-Lizenz (CC BY) versehen. Dies erlaubt Benutzerlnnen, die Karten frei zu nutzen, zu teilen und bei Bedarf auch zu verändern. Die zugrunde liegenden Daten, also die von Open Knowledge Maps generierten Datenstrukturen, sind in der Public Domain (CCO).

Open Knowledge Maps sieht sich als Baustein der offenen Infrastruktur und arbeitet auch aktiv mit anderen Anbietern zusammen. Dazu gehören BASE als Standard-Datenbank, rOpenSci, welche die Datenclients für den einfachen Zugriff auf Datenquellen bereitstellen, oder Hypothesis, dessen Annotations-Service in die Wissenslandkarten integriert ist. Als eine Konsequenz des offenen Ansatzes kann die Software auch auf andere Datenquellen und Verbundinfrastrukturen wie bspw. die European Open Science Cloud (EOSC) angewendet werden. Ein Beispiel dafür ist das Projekt VIPER (Kraker et al. 2018), welches in Zusammenarbeit mit OpenAIRE entwickelt wurde. Basierend auf der gleichen Open Source-Software wie Open Knowledge Maps bietet VIPER (kurz für "Visual Project Explorer") einen visuellen Überblick über die in OpenAIRE indizierten Projekte. VIPER ermöglicht damit Fördergebern, Institutionen, Forschenden und anderen Stakeholdern, die Ergebnisse von Projekten systematisch zu untersuchen und deren Rezeption in verschiedenen Bereichen zu verstehen.

Dieser offene Ansatz endet nicht bei der Technologie. Ziel von Open Knowledge Maps ist es, eine nachhaltige und inklusive Plattform für alle diejenigen zu schaffen, die von wissenschaftlichem Wissen profitieren können. Um dieses Ziel zu erreichen, arbeiten wir mit Menschen und Organisationen zusammen, die sich für bessere und offene Lösungen für die Auffindbarkeit von Wissen einsetzen. Dazu wurde unter anderem ein 
Community Outreach-Programm, das "Enthusiasts-Programm"4, gestartet. Bisher haben die Enthusiasts Veranstaltungen auf vier Kontinenten mit zusammen mehr als 100 Teilnehmerlnnen durchgeführt. Sie haben dabei nicht nur zur Verbreitung von Techniken zur Literatursuche und dem Wissen über innovative Tools beigetragen, sondern auch wertvolles Feedback zu Open Knowledge Maps gesammelt. Dieses Feedback fließt wiederum in die Weiterentwicklung von Open Knowledge Maps ein.

Um Open Knowledge Maps hat sich so in den letzten drei Jahren eine begeisterte Gemeinschaft gebildet. Wir konnten mehr als eine halbe Million Besuche aus der ganzen Welt auf der Website verzeichnen, die mehr als einhunderttausend Karten erstellt haben. Die meisten NutzerInnen kommen dabei aus Indonesien, gefolgt von den USA, Indien, Deutschland, Österreich, UK, China, Kanada und Australien. Zudem wurden in Workshops und Seminaren über 1.500 Teilnehmerlnnen in innovativen Techniken zur Literatursuche geschult ${ }^{5}$.

All dies macht Open Knowledge Maps zu einer Infrastruktur, die durch die wissenschaftliche Community vorangetrieben wird und sich gleichermaßen in ihrem Eigentum befindet. So wird außerdem sichergestellt, dass bei der Weiterentwicklung von Open Knowledge Maps die Anforderungen der Benutzerlnnen berücksichtigt werden.

\section{Aus Österreich für die ganze Welt}

Open Knowledge Maps ist weltweit tätig, hat jedoch einen starken Österreich-Bezug. Die gemeinnützige Organisation ist in Wien registriert und hat hier auch ihr Hauptquartier. Gründer und Obmann Peter Kraker legte die Grundlagen für Open Knowledge Maps in seiner Zeit als Dissertant und Postdoc am Forschungszentrum Know-Center an der TU Graz. Das Know-Center ist auch organisationales Mitglied des Teams von Open Knowledge Maps und unterstützt den Verein tatkräftig. Die Ludwig Boltzmann Gesellschaft (LBG) ist seit 2019 als erstes Fördermitglied an Bord. Mit dem Open Innovation in Science Center der LBG führt Open Knowledge Maps auch Kollaborationsprojekte durch; unter anderem wurde eine Visualisierung der Ergebnisse des wegweisenden Crowdsourcing-Projektes CRIS I erstellt ${ }^{6}$.

Mit der Österreichischen Akademie der Wissenschaften führt Open Knowledge Maps das Projekt LinkedCat+ durch. Dabei werden die Sitzungsberichte der ÖAW von 1847-1918 zugreifbar, auffindbar und nachnutzbar gemacht (Details siehe Abschnitt „Starke Partnerschaft mit Bibli- 
otheken“). Zudem ist Open Knowledge Maps Mitglied im Open Science Network Austria (OANA) und bei „Österreich forscht!“ und pflegt Partnerschaften mit Wikimedia Österreich und WeBuildEurope.

\section{Nachhaltigkeit durch ein konsortiales Fördermodell}

Im nächsten Schritt soll der partizipative Ansatz von Open Knowledge Maps mit einem konsortialen Fördermodell kombiniert werden. Damit soll ein entscheidender Schwachpunkt behoben werden, die nachhaltige Finanzierung der Organisation. Die bisherigen Meilensteine wurden mit einem vergleichsweise winzigen Budget erreicht - dem zehnköpfigen Team stehen Mittel für gerade einmal ein Vollzeitäquivalent zur Verfügung. Open Knowledge Maps ist nur dank tausender Stunden, die von enthusiastischen Ehrenamtlichen im Team eingebracht wurden, entstanden. Es ist offensichtlich, dass dies kein nachhaltiges Vorgehen ist und dass das Weiterentwicklungspotenzial eines Projekts, welches rein auf ehrenamtlicher Arbeit basiert, begrenzt ist.

In dem nun angestrebten konsortialen Fördermodell, das dem der Open Library of Humanities (OLH) und dem des Directory of Open Access Journals (DOAJ) entspricht, werden Organisationen Fördermitglieder von Open Knowledge Maps und steuern einen jährlichen Mitgliedsbeitrag ${ }^{7}$ bei. Im Gegenzug lädt Open Knowledge Maps die Fördermitglieder ein, die Plattform mitzugestalten. Konkret können sie über Funktionen und Datenquellen abstimmen, die in Open Knowledge Maps integriert werden sollen und haben so ein Mitspracherecht bei der Erstellung des jährlichen Entwicklungsplans.

\section{Exkurs: die problematische Fördersituation gemeinnütziger Open Source-Initiativen}

Insgesamt sind die Funding-Möglichkeiten für gemeinnützige Organisationen und Open Source-Projekte stark eingeschränkt. Besonders für Frontend-Services steht wenig Geld zur Verfügung. Dadurch wird allerdings Kontrolle und Governance über die Art und Weise, wie Forschende und andere Stakeholder-Gruppen mit Wissenschaft interagieren, an kommerzielle Unternehmen abgetreten. Diese haben vorrangig den Auftrag, Shareholder Value zu maximieren und handeln daher nicht immer im Interesse der wissenschaftlichen Community und der Gesellschaft. Auch die Nutzungsdaten verbleiben in 
weiterer Folge bei diesen For-Profits, was zu Problemen führen kann, wenn diese Daten weitergegeben oder verkauft werden. Zudem kommt es bei proprietären Lösungen oft zu Lock-In-Effekten, d.h. dass ein Umstieg auf ein anderes System mit erheblichen Kosten verbunden wäre. Dadurch können die Lizenzgebühren jährlich oft weit über die Inflation erhöht werden. Eine Situation, die jenen sehr bekannt vorkommen dürfte, die für eine Umstellung von Closed Access zu Open Access bei Publikationen eintreten.

Literatursuchsysteme rein proprietären und geschlossenen Lösungen zu überlassen beschränkt aber auch Innovation, da es in einem Markt mit einigen wenigen Anbietern wenige Anreize zur Verbesserung des eigenen Angebots gibt. Dies war in der Vergangenheit bei den Anbietern zu beobachten. Man hat sich vielmehr darauf konzentriert, innovative Mitbewerber durch hohe Eintrittsbarrieren (Aufbau eines eigenen Indexes) vom Markteintritt abzuhalten. Im Gegensatz dazu gedeihen Innovationen in einer offenen Discovery-Infrastruktur. Wie in Abbildung 6 zu sehen, können die Bausteine der Infrastruktur aufeinander aufbauen und setzen so einen sich selbst verstärkenden, positiven Kreislauf in Gang. Es gibt auch keine Lock-In-Effekte, wie sie bei geschlossenen Angeboten auftreten. Wenn sich eine Organisation nicht so verhält, wie es die Community erwartet, kann die Community zu einer Alternative wechseln oder selbst eine Plattform basierend auf der Open Source-Software aufsetzen. Offene Infrastrukturen sind daher die stärksten Innovationstreiber für wissenschaftliche Infrastrukturen.

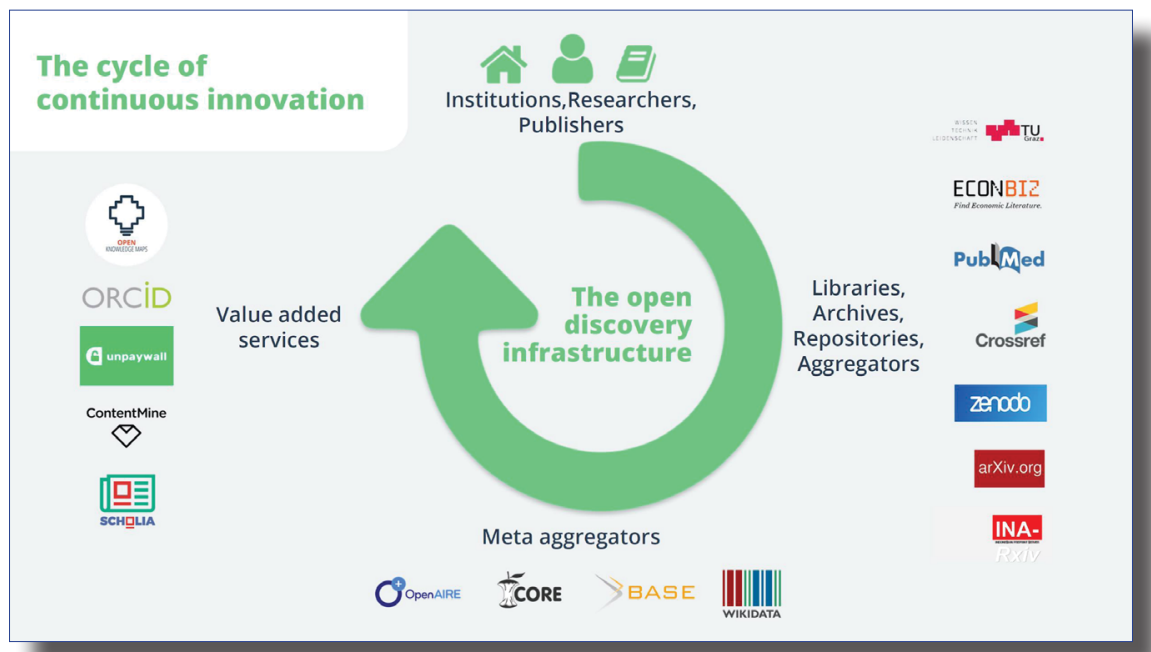

Abb. 6: Die offene Discovery-Infrastruktur 
Die Krise der Auffindbarkeit wurde durch proprietäre Systeme ausgelöst und kann nur durch Investitionen in offene Infrastrukturen und offene Frontends bekämpft werden. Dadurch können die Früchte von Open Access geerntet und Dark Knowledge wieder sichtbar werden. Mehr Informationen dazu finden Sie im Rahmen der \#DontLeaveltToGoogle-Kampagne, welche von Open Knowledge Maps-Gründer Peter Kraker (siehe u.a. Kraker 2018) initiiert wurde.

\section{Starke Partnerschaft mit Bibliotheken}

Bibliotheken ermöglichen seit Jahrtausenden die Auffindbarkeit wissenschaftlicher Erkenntnisse und BibliothekarInnen sind ExpertInnen in der Beschaffung, Kuratierung und Verbreitung von Forschung. Daher hat Open Knowledge Maps starke Partnerschaften und eine enge Zusammenarbeit mit Bibliotheken etabliert. Namhafte Bibliothekarlnnen aus dem In- und Ausland zählen zum Advisory Board und viele begleiten Open Knowledge Maps von Beginn an ${ }^{8}$.

Mit BASE als Hauptdatenquelle baut Open Knowledge Maps auf Bibliotheksinfrastrukturen auf und erhöht die Sichtbarkeit der darin enthaltenen Inhalte z.B. aus Universitätsrepositorien. Dazu gehören viele Quellen die von kommerziellen Produkten nicht indiziert werden. Mit Open Knowledge Maps kann auch eine Vielzahl von Dokumenttypen durchsucht werden, einschließlich Datensätze, Bilder und Multimediainhalte, wodurch sich der Dienst auch für Spezialsammlungen eignet, die von Bibliotheken in großer Zahl verwaltet werden.

Darüber hinaus arbeitet Open Knowledge Maps mit Bibliotheken in der Entwicklung innovativer Open Source-Projekte zusammen. Dazu zählt das Projekt LinkedCat+, welches von der Österreichischen Akademie der Wissenschaften gefördert und von der ÖAW BAS:IS (Bibliotheken, Archive, Sammlungen) geleitet wird. Ziel dieses Projekts, welches auch in Zusammenarbeit mit dem Austrian Centre for Digital Humanities $(A C D H)$ durchgeführt wird, ist es, die Sitzungsberichte der ÖAW von 1847-1918 zugreifbar, auffindbar und nachnutzbar zu machen. Die Sitzungsberichte werden dabei als Open Access-Volltexte zur Verfügung stehen und die bibliographischen Daten als Linked Open Data. Open Knowledge Maps entwickelt das Discovery-Frontend, welches neben Wissenslandkarten auch eine autorenbasierte Visualisierung und einen Zeitstrahl beinhaltet und so einen umfassenden Blick auf das Material ermöglicht. 
Bibliotheken sind wichtige Treiber von Open Science und sie sind an vielen Initiativen zur Nachhaltigkeit gemeinnütziger Open Science-Infrastruktur beteiligt. Open Knowledge Maps lädt die Bibliotheken nun ein, die Plattform im Rahmen des konsortialen Finanzierungsmodells gemeinsam zu gestalten und die Zusammenarbeit so zu verstetigen.

\section{Ausblick: Kollaborative Literaturrecherche}

In Zukunft wird sich Open Knowledge Maps unter anderem dem wichtigen Thema der besseren Auffindbarkeit von Datensätzen widmen. Dazu wurde mit 19 anderen Organisationen (u.a. CESSDA, DataCite, EUDAT und OpenAIRE) ein eigenes GO FAIR-Implementierungsnetzwerk gegründet, welches im März 2019 die Arbeit aufgenommen hat.

Zudem will Open Knowledge Maps kollaborative Literaturrecherche ermöglichen. Derzeit wird zum überwiegenden Teil alleine und immer wieder von neuem recherchiert. Das dabei gewonnene Wissen verbleibt aber zumeist in den Köpfen und kann nur schwer in strukturierter Form weitergegeben werden. Open Knowledge Maps soll nun dahingehend weiterentwickelt werden, dass Wissenslandkarten verändert, erweitert und anschließend wieder geteilt werden können. Dadurch kann man bei der Recherche auf existierendes Wissen aufbauen und muss nicht bei Null beginnen. Diese Daten werden in weiterer Folge auch über Datenschnittstellen zur Verfügung stehen, sodass sie auch in anderen Angeboten genutzt werden können.

Auch hier sehen wir Bibliotheken und Bibliothekarlnnen als wichtige PartnerInnen. Ein kollaboratives System kann nicht ohne die ExpertInnen in den Bereichen Wissenskuratierung, -strukturierung und -verwaltung funktionieren. Gemeinsam mit anderen Stakeholdern aus Wissenschaft und Gesellschaft soll so ein System entstehen, welches den Einstieg in wissenschaftliches Wissen ermöglicht und diesen intellektuellen Schatz für alle nutzbar macht. 
Dr. Peter Kraker

ORCID: https://orcid.org/0000-0002-5238-4195

Open Knowledge Maps E-Mail: pkraker@openknowledgemaps.org

Maxi Schramm

Open Knowledge Maps

E-Mail: maxi@openknowledgemaps.org

Christopher Kittel, B.A. B.Sc. ORCID: https://orcid.org/0000-0002-1894-5040

Open Knowledge Maps E-Mail: christopher.kittel@openknowledgemaps.org

\section{Danksagung}

Wir danken dem Projektteam der FH Joanneum Graz, Benjamin Barteder, Sandrine Fackner und Sonja Radkohl, sowie Betreuer Robert Gutounig für die Zusammenarbeit bei der Erstellung des Fragebogens und der Durchführung der Umfrage.

\section{Literatur}

Börner, Katy, Chaomei Chen, and Kevin W. Boyack (2003). Visualizing knowledge domains. Annual Review of Information Science and Technology 37(1), 179-255. https://doi.org/10.1002/aris.1440370106

Brownson, Ross C., Matthew W. Kreuter, Barbara A. Arrington, and William R. True (2006). From the Schools of Public Health'. Public Health Reports 121(1), 97-103. https://doi.org/10/gfkr2s

Bornmann, Lutz, and Rüdiger Mutz (2015). Growth Rates of Modern Science: A Bibliometric Analysis Based on the Number of Publications and Cited References: Growth Rates of Modern Science: A Bibliometric Analysis Based on the Number of Publications and Cited References. Journal of the Association for Information Science and Technology 66(11), 2215-2222. https://doi.org/10.1002/asi.23329

Dahn, Bernice, Vera Mussah, and Cameron Nutt. (2015). Opinion | Yes, We Were Warned About Ebola. The New York Times, 7 April, sec. Opinion. https://www.nytimes.com/2015/04/08/opinion/yes-we-were-warnedabout-ebola.html 
Jeschke, Jonathan, Sophie Lokatis, Isabelle Bartram, and Klement Tockner (2019). Knowledge in the Dark: Scientific Challenges and Ways Forward. FACETS 4, 423-441. https://doi.org/10.1139/facets-2019-0007 Johnson, Rob, Anthony Watkinson, and Michael Mabe (2018). The STM Report: An overview of scientific and scholarly publishing. https:// www.stm-assoc.org/2018_10_04_STM_Report_2018.pdf

Kraker, Peter, Maxi Schramm, Christopher Kittel, Scott Chamberlain, and Thomas Arrow (2018). VIPER: The Visual Project Explorer. Zenodo. https://doi.org/10.5281/zenodo.1248119

Kraker, Peter, Christopher Kittel, and Asura Enkhbayar (2016). Open Knowledge Maps: Creating a Visual Interface to the World's Scientific Knowledge Based on Natural Language Processing. 027.7 Zeitschrift für Bibliothekskultur / Journal for Library Culture 4(2), 98-103. https://doi.org/10.12685/027.7-4-2-157

Kraker, Peter, Christopher Kittel, Maxi Schramm, Rainer Bachleitner, Thomas Arrow, Scott Chamberlain, Asura Enkhbayar, et al. (2019). Headstart 5. Zenodo. https://doi.org/10.5281/zenodo.2587129

Kraker, Peter (2018). Google Is Capitalizing on a Movement That They Have Contributed Nothing To. Elephant in the Lab (September 27). https://doi.org/10.5281/zenodo.1434695

Kraker, Peter, Christian Schlögl, Kris Jack, and Stefanie Lindstaedt (2015). Visualization of Co-Readership Patterns from an Online Reference Management System. Journal of Informetrics 9(1), 169-182. https://doi. org/10.1016/j.joi.2014.12.003 (Author Accepted Manuscript verfügbar unter: https://arxiv.org/abs/1409.0348)

Nicolaisen, Jeppe, and Tove Faber Frandsen (2019). Zero Impact: A LargeScale Study of Uncitedness. Scientometrics 119(2), 1227-1254. https://doi.org/10.1007/s11192-019-03064-5

Peters, Isabella, Peter Kraker, Elisabeth Lex, Christian Gumpenberger, and Juan Gorraiz (2016). Research Data Explored: An Extended Analysis of Citations and Altmetrics. Scientometrics 107(2), 723-744. https://doi. org/10.1007/s11192-016-1887-4

Shneiderman, Ben (1996). The Eyes Have It: A Task by Data Type Taxonomy for Information Visualizations. Proceedings of the IEEE Symposium on Visual Languages (Boulder, CO, USA), 336-343. https://doi. org/10.1109/VL.1996.545307 (Frei zugänglich unter: https://www. cs.umd.edu/ ben/papers/Shneiderman1996eyes.pdf)

"Ebolafieber-Epidemie 2014." In Wikipedia, May 2, 2019. https://de.wikipedia.org/w/index.php?title=EbolafieberEpidemie_2014\&oldid=188146618 
1 https://www.research4life.org/access/

2 Siehe https://openknowledgemaps.org/team

3 Hierbei wird der Relevanz-Suche von BASE bzw. PubMed genutzt. Dabei handelt es sich um die Dokumente mit der größten textuellen Ähnlichkeit zwischen Suchbegriff und den Metadaten der Dokumente.

4 Siehe https://openknowledgemaps.org/community

5 Die Workshop-Materialien stehen ebenfalls frei zur Verfügung, siehe http://openknowledgemaps.org/community\#training-materials

6 Siehe https://ois.lbg.ac.at/en/cris-I-research-questions

7 Für eine Übersicht siehe https://openknowledgemaps.org/supportingmembership

8 Siehe https://openknowledgemaps.org/team\#advisory-board 